\title{
High-efficiency WLANs for dense deployment scenarios
}

\author{
B T VIJAY* ${ }^{*}$ and B MALARKODI
}

\author{
Department of Electronics and Communication Engineering, National Institute of Technology, \\ Tiruchirappalli 620 015, India \\ e-mail: bt.vvts@gmail.com; malark@nitt.edu
}

MS received 22 March 2018; revised 8 August 2018; accepted 9 August 2018; published online 25 January 2019

\begin{abstract}
In this article, we review the latest technical attributes such as orthogonal frequency division multiple access (OFDMA), multi-user MIMO (MU-MIMO) and enhanced clear channel assessment (CCA) for better spatial reuse used in the 802.11ax amendment to the 802.11 standard that leads to PHY and MAC enhancements for high-density scenarios of access points (APs). IEEE 802.11ax, also referred to as highefficiency wireless local area network (WLAN) (HEW), provides mechanisms to thoroughly utilize the unlicensed spectrum bands $(2.4$ and $5 \mathrm{GHz})$ and strengthen the user experience. The functional requirements of $\mathrm{HEW}$ are stressed on interactive video transmission latency and access efficiency to meet quality of service (QoS) requirements. Finally, we investigate three configurations-MU-MIMO, OFDMA and combination of both or mixed mode-for 4-user AP transmission schemes in 802.11ax. The performance of the MU schemes varies with packet size and operating SNR. OFDMA is more efficient than MU-MIMO at low SNRs for all packet sizes, which means 5th percentile stations (STAs) can get desired throughput.
\end{abstract}

Keywords. CCA; high-efficiency WLAN (HEW); spatial reuse; MAC; multi-user (MU); OFDMA; throughput; 802.11ax; efficiency.

\section{Introduction}

The use of IEEE 802.11-based wireless local area networks (WLANs) has more than doubled in recent times due to its ability to provide increased mobility and simplicity, with reduced cost of installation and maintenance. It has triggered massive WLAN deployment in geographically restricted environments that involve multiple overlapping basic service sets (OBSSs). Since its first release in 1997, the standard defines the MAC procedures to support local area network (LAN) applications with quality of service (QoS) requirements, including the transport of voice, audio and video (AV). The standard describes MAC mechanisms to support the prioritization of management frames, and specifies mechanisms to improve AV streaming QoS while maintaining data and voice performance [1]. The recently approved standard IEEE 802.11ac-2013 enables very high throughput (VHT) greater than $1 \mathrm{Gbps}$. The most significant PHY layer upgrades are the $80-\mathrm{MHz}$ channel access and $160 \mathrm{MHz}(80+80)$ combination approach, $8 \times 8$ MIMO antenna service and downlink multi-user MIMO (DL MU-MIMO), which can be useful to permit aggregated frames to be sent from the access point (AP) to many receivers through multiple spatial streams [2-4]. A critical MAC layer enhancement is TXOP sharing, which can be

*For correspondence useful to handle quite a few downlink traffic streams to many receiver stations (STAs) concurrently. WLAN interfaces are being implemented in more and more products such as personal computers and smart phones connections, resulting in rapid growth of the market for Wi-Fi-enabled terminals with over 12 billion cumulative shipments from 2015 onwards [2, 5, 6]. However, new problems have emerged with the proliferation of WLAN connections. One problem is degradation of transmission efficiency, which occurs when many Wi-Fi terminals interfere with each other. The cause of this is that WLAN devices acquire channel access opportunities under the Carrier Sense Multiple Access with Collision Avoidance (CSMA/CA) MAC protocol, which avoids simultaneous transmission on the same frequency resource; this means that the transmission opportunities of each terminal decrease in dense deployment scenarios. Also, users utilize WLAN for many different applications such as video streaming and offloading.

A new study group called high-efficiency WLAN (HEW): IEEE 802.11ax to enhance 802.11 PHY and MAC in 2.4 and $5 \mathrm{GHz}$ unlicensed bands was formed recently. HEW focused on improving spectrum efficiency, area throughput and real-world performance in both indoor and outdoor deployments in the presence of interfering sources, dense heterogeneous networks and in moderate-heavy user-loaded APs. Task Group 802.11ax (TGax) is an advanced version of WLAN in the set of WLAN Standards, 
and it is a successor to IEEE 802.11ac. IEEE 802.11ax is predicted to have a maximum capacity of around $10 \mathrm{Gbps}$ by utilizing orthogonal frequency division multiple access (OFDMA) PHY, AP-initiated trigger frame, spatial reuse (SR) and multi-user MIMO (MU-MIMO) supporting both uplinks as well as downlinks, which delivers 4 times the throughput of IEEE 802.11ac; aggregation mechanism for ACK/BA is still in the mid-stages of development, expected to be accomplished in 2019 [7].

In the following sections, first we discuss the functional requirements for IEEE 802.11ax. Second, we introduce the high-efficiency PHY enhancements and briefly explain MU aggregation and HE channel sounding protocol; third, we talk about new features of highefficiency MAC, mainly covering SR operation using enhanced clear channel assessment (CCA) by dynamic sensitivity control (DSC) with colour codes and opportunistic adaptive CCA (OA-CCA); in the same section, we discuss the target wake time (TWT) operation and access efficiency requirements for HEW. Lastly, we present the AP throughput analysis with the help of three configurations-MU-MIMO, OFDMA and combination of both or mixed mode-for DL MU transmission in IEEE 802.11ax, followed by the conclusion.

\section{Functional requirements}

There have been research works in the HEW study group for functional requirements that highlight essential scope concepts of 802.11ax (HEW). (a) System performance: the 802.11ax amendment shall provide at least one mode of operation capable of achieving at least four times improvement in the average throughput per STA (measured at the MAC data service AP) in a dense deployment scenario while maintaining or improving the power efficiency per STA. The HEW shall bring latency to a desirable level to meet QoS requirements in a high-density deployment scenario, which is discussed briefly in the next subsection. (b) Spectrum efficiency: the 802.11ax amendment shall provide a mechanism to facilitate efficient use of spectrum resources and to manage interference between neighbouring OBSS in a dense deployment scenario. The 802.11ax amendment shall provide a mechanism to increase the spectral efficiency of $20-\mathrm{MHz}$ and larger channels in scenarios with a high density of HEW STAs and scenarios with the presence of legacy devices. The 802.11 ax amendment may provide a mechanism to increase frequency reuse efficiency and manage interference in the deployment scenarios with a high density of HEW STAs and with the presence of transmission between non-AP STAs. (c) Bands of operation: define bands of operations in frequency bands between 1 and $6 \mathrm{GHz}$. (d) Backward compatibility and coexistence: legacy performance shall not be significantly degraded by operation in or in proximity to a HEW network. Finally, the 802.11ax amendment shall define mechanisms to improve the 5th percentile of per STA throughput without degradation of afore-listed performance requirements.

Interactive video transmission latency requirement: The mechanisms defined in the 802.11ax amendment shall significantly improve the average transmission latency of latency-sensitive applications to satisfy QoS requirements when compared with the active IEEE 802.11 standard and its amendments operating in the same band (IEEE $802.11 \mathrm{n}$ in $2.4 \mathrm{GHz}$ and IEEE $802.11 \mathrm{ac}$ in $5 \mathrm{GHz}$ ) and the same deployment scenario. The transmission latency is measured from the time that the MPDU is set for transmission (i.e., beginning of CSMA/CA access); MAC receives a packet till the time that PHY begins transmitting. It is clear that the video traffic will continue to rise and will become the dominating traffic type for WLAN in the current situation. HD TVs and the popularity of video casting from/to mobile devices are defining the user experience by the quality of a wireless video [7]. PER is a primary QoS metric. Along with PER, video traffic uses WLAN packet latency and jitter as added metrics for QoS. Since HEW needs to provide $\mathrm{QoS}$ for high-density video traffic, HEW functional requirements should also address video packet delay across the WLAN.

HEW needs packet latency requirement: For most streaming video services (e-education, Netflix, etc.), sizeable video buffering at the display is used to combat jitter and adds 1-10 s end-to-end delay. Packet delay is only a small portion of total delay, and even high delays $(10-50 \mathrm{~ms})$ are regarded as tolerable. However, for interactive video, wireless virtual desktop and other video applications, end-to-end delay is not acceptable. HEW must define a packet latency requirement that will address the needs of next generation users using delay-sensitive applications. The WLAN packet delay must result from an endto-end delay plan for interactive video applications. The WLAN portion of the end-to-end delay should be an added functional requirement for HEW just for delay-sensitive applications [7-9].

\section{High-efficiency PHY}

A high-efficiency device will be asked to keep essential requirements of the legacy WLAN PHY layers. This could be a HE device operating at $2.4 \mathrm{GHz}$ having to meet the legacy PHY requirements; a HE device operating in the $5 \mathrm{GHz}$ band will have to work with the legacy and VHT PHY specifications. Despite this submission requirement, there are vital improvements in 802.11ax from past 802.11 generations. Several variations are the inclusion of service designed for MU-MIMO in the uplink and downlink along with OFDMA. Further, Modify in dot11ax has the symbol time of $12.8 \mu \mathrm{s}$, which is four times the legacy symbol time 
Table 1. Overview comparison of dot $11 \mathrm{n}$, dot11ac and dot11ax.

\begin{tabular}{|c|c|c|c|}
\hline & IEEE $802.11 \mathrm{n}$ & IEEE 802.11ac & IEEE 802.11ax \\
\hline $\begin{array}{l}\text { Frequency bands } \\
\quad(\mathrm{GHz})\end{array}$ & $2.4,5$ & 5 & $2.4,5$ \\
\hline $\begin{array}{l}\text { Channel } \\
\text { bandwidth } \\
(\mathrm{MHz})\end{array}$ & 20,40 & $20,40,80,80+80,160$ & $20,40,80,80+80,160$ \\
\hline $\begin{array}{l}\text { Subcarrier } \\
\quad \text { spacing }(\mathrm{kHz})\end{array}$ & 312.5 & 312.5 & 78.125 \\
\hline Symbol time $(\mu \mathrm{s})$ & 3.2 & 3.2 & 12.8 \\
\hline Cyclic prefix $(\mu s)$ & 0.8 & $0.8,0.4$ & $0.8,1.6,3.2$ \\
\hline Multi-user (MU) & No & Only downlink & $\begin{array}{c}\text { AP-initiated uplink Tx plus downlink } \\
\text { Tx }\end{array}$ \\
\hline Modulation & OFDM & OFDM & OFDM, OFDMA \\
\hline $\begin{array}{l}\text { Data subscriber } \\
\text { modulation }\end{array}$ & BPSK, QPSK, 16-QAM, 64-QAM & $\begin{array}{c}\text { BPSK, QPSK, 16-QAM, 64-QAM, } \\
\text { 256-QAM }\end{array}$ & $\begin{array}{c}\text { BPSK, QPSK, 16-QAM, 64-QAM, } \\
\text { 256-QAM, 1024-QAM }\end{array}$ \\
\hline Coding & BCC (mandatory) LDPC (optional) & BCC (mandatory) LDPC (optional) & BCC (mandatory) LDPC (mandatory) \\
\hline Data rates & $\begin{array}{l}\text { 72.2 Mbps }(20 \mathrm{MHz}, 1 \mathrm{SS}) \\
600 \mathrm{Mbps}(40 \mathrm{MHz}, 4 \mathrm{SS})\end{array}$ & $\begin{array}{l}433 \mathrm{Mbps}(80 \mathrm{MHz}, 1 \mathrm{SS}) \\
6.93 \mathrm{Gbps}(160 \mathrm{MHz}, 8 \mathrm{SS})\end{array}$ & $\begin{array}{l}\text { 600.4 Mbps (80 MHz, 1 SS) } \\
9.6078 \mathrm{Gbps}(160 \mathrm{MHz}, 8 \mathrm{SS})\end{array}$ \\
\hline
\end{tabular}

of $3.2 \mu \mathrm{s}$. Three major reasons concerning increase of the symbol time are as follows [8, 10]: (i) robustness in outdoor channels, (ii) better tolerance to timing jitter across users in UL MU-MIMO/OFDMA and (iii) better indoor efficiency by cutting down cyclic prefix (CP) overhead. Along with the increased symbol time, dot11ax requires support for three CPs time [8-11] (table 1): (a) $0.8 \mu \mathrm{s}$-legacy CP time with the longer symbol time improves efficiency due to reduced overhead from the $\mathrm{CP}$, (b) $1.6 \mu \mathrm{s}$-focusing on high efficiency in outdoor channels and indoor UL MUMIMO/OFDMA and (c) $3.2 \mu \mathrm{s}$-concentrate on robustness in the more appropriate case of outdoor UL MU-MIMO/ OFDMA.

As an effect of the longer symbol time, the subcarrier spacing reduces from 312.5 to $78.125 \mathrm{kHz}$, and the FFT size for a channel bandwidth of $20 \mathrm{MHz}$ grows from 64 to 256. Narrow subcarrier spacing helps improved equalization, and thereby a better channel robustness. However, the implementation complexities increase as FFT grows. Also, there are two new MCSs, 1024 QAM 3/4 and 1024 QAM 5/6, denoted as MCS10 and MCS11, respectively, applicable for channels with a bandwidth larger than $20 \mathrm{MHz}$; also, as the subcarrier spacing becomes smaller, the CFO (carrier frequency offset) correction is required to be more appropriate. Figure 1a shows the new HE PPDU formats and table 2 provides the acronym decoder ring for the fields used in the PPDUs [12].

SU and MU formats: Currently two types of preambles (SU format and MU format) are defined with differentiation on whether SIGB is transmitted or not. While the MU format carries the most general OFDMA + MU-MIMO packets, the SU format provides efficiency for single-user transmissions. Both preambles can be used by any device for transmission in the UL, DL or peer to peer. Both preambles are essential for $11 \mathrm{ax}$ and we therefore propose that both shall be mandatory

Legacy preamble: To keep backward compatibility with legacy devices $(11 \mathrm{a} / \mathrm{n} / \mathrm{ac})$, it is straightforward to prepend a legacy preamble before each 11ax PPDU; dot11ax receiver can initially acquire synchronization through L-STF and L-LTF, and channel estimation to decode $11 \mathrm{ax}$ preamble. Legacy STA can detect the PPDU existence and defer their transmission by the length indicated in L-SIG. It is more reliable $(-82 \mathrm{dBm})$ and energy efficient than ED to prevent the unexpected interference from legacy STA [12].

HE-SIG-A: All intended 11n/ac receivers decode HTSIG/VHT-SIG-A following legacy preamble to obtain common configuration information of the following PPDU; 11ax PPDU should also contain HE-SIG-A to carry some similar fields as in HT-SIG/VHT-SIG-A of $11 \mathrm{n} / \mathrm{ac}$, e.g., BW and GI. HE-SIG-A uses 64-FFT on each 20-MHz channel, and receiver can perform coherent detection of HE-SIG-A based on the CSI estimation from L-LTF. Legacy STA can be spoofed to receive an 11a/ac PPDU by specific design of modulation scheme. Like $11 \mathrm{n} / 11 \mathrm{ac}$, the legacy preamble and HE-SIG-A shall be duplicated on each $20 \mathrm{MHz}$ in order to maintain the reliability of carrier sensing and low PAPR at transmitter.

802.11ax is the first WLAN standard to use OFDMA to enable transmissions with multiple users at the same time. This technology has been used in other standards, including WiMAX and LTE, and is also being used in 5G NR. With OFDMA, the bandwidth is divided into resource units (RUs) of various sizes, and different numbers and sizes of RUs can be allocated to transmissions to different users, 

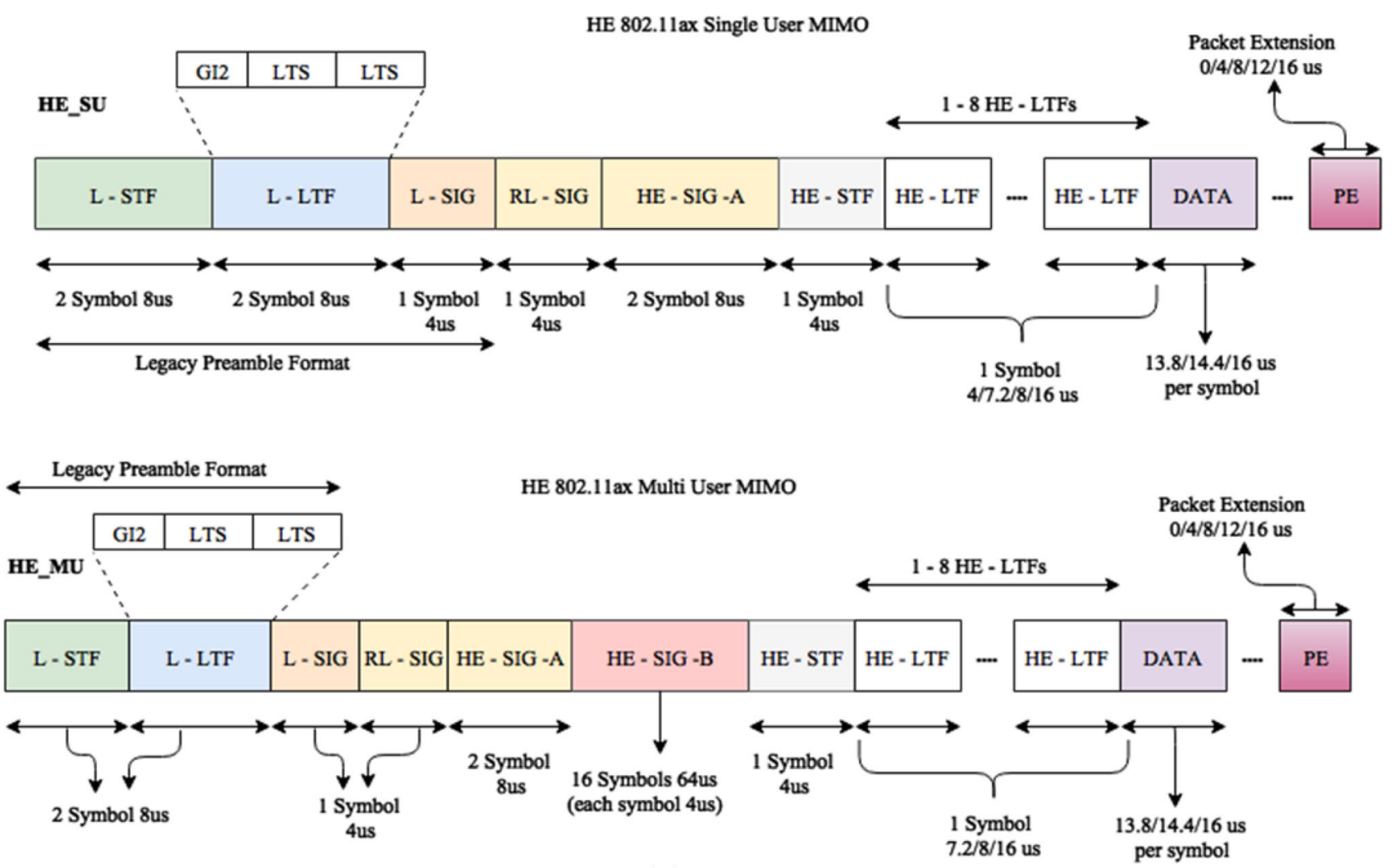

(a)

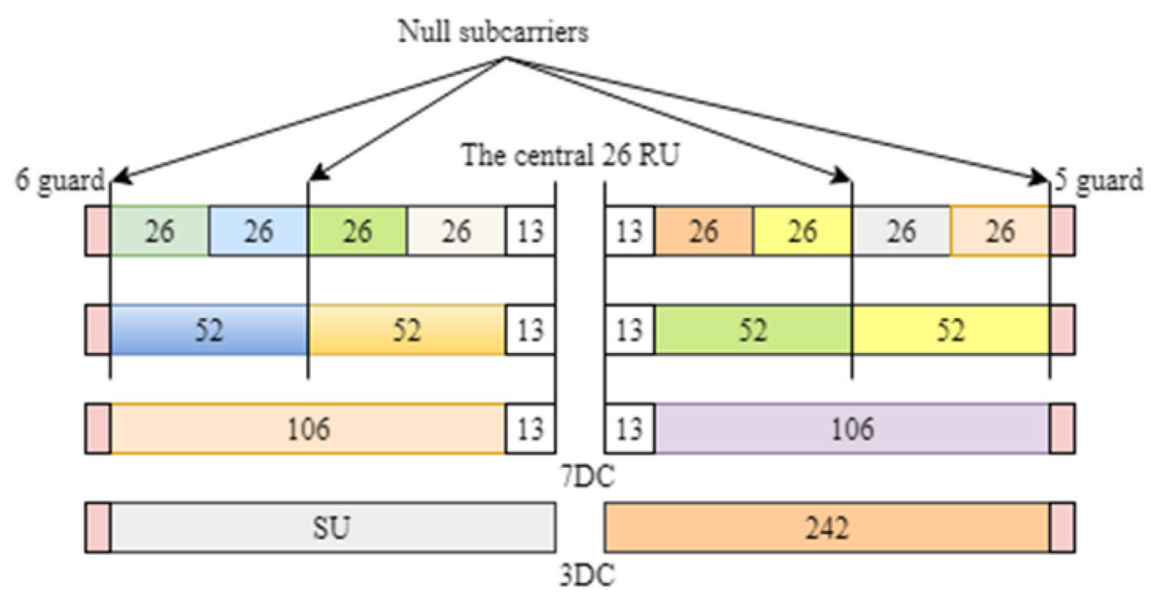

(b)

Figure 1. (a) HE PPDU formats. (b) RU locations in a 20-MHz HE PPDU.

based on the data throughput requirements [12-15]. The size of an RU can be 26, 52, 106, 242, 484, 996 or $2 \times 996$ tones or subcarriers, and the location of the RUs is defined for $20-, 40-$ and $80-\mathrm{MHz}$ channels. The $20-\mathrm{MHz} \mathrm{RU}$ structure is used for each $20-\mathrm{MHz}$ segment of one $40-\mathrm{MHz}$ transmission. Figure $1 \mathrm{~b}$ shows the location of RUs for a 20-MHz channel [16].

\subsection{MU aggregation}

In legacy WLAN, different access category (AC) traffic cannot be transmitted in an A-MPDU for contention fairness consideration. In high-efficiency case OFDMA/MUMIMO, the airtime of scheduled DL/UL OFDMA RU is set by the STA that requires the longest airtime. Remaining STAs will and may have long idle time. Allowing AP or 
Table 2. PPDU field representation.

\begin{tabular}{lc}
\hline Field & Representation \\
\hline L-STF & Legacy short training field \\
L-LTF & Legacy long training field \\
L-SIG & Legacy signal field \\
RL-SIG & Repeated legacy signal field \\
HE-SIG-A & HE signal A field \\
HE-SIG-B & HE signal B field \\
HE-STF & HE short training field \\
HE-LTF & HE long training field \\
PE & Packet extension field \\
GI & Guard interval \\
LTS & Legacy training sequence \\
\hline
\end{tabular}

STA to aggregate frames from different ACs will improve the utilization of the scheduled RUs. In DL OFDMA/MUMIMO, AP aggregates frames from secondary ACs of the same STA, whereas in UL OFDMA/MU-MIMO, STA aggregates frames from secondary ACs. The VHT-SIG-B length field for each user indicates the number of octets, rounded to the next 4 octet boundary, in the A-MPDU excluding padding. This value allows the PHY to stop processing receive once all the useful MAC data have been received [12].

When compared with LTE, WLAN does not have mandatory MU aggregation mechanisms and is less suited for short packets. Considering an application with a high likelihood of short packets/bursts, MU aggregation mechanisms help enhance MAC efficiency and reduce medium access overhead mainly in dense environments. Another prime requirement of MU aggregation is to have efficient aggregation for ACK/BA. Although such aggregation can be made available in DL with some specification changes except for UL direction, there is no efficient ACK aggregation mechanism. If UL OFDMA gets adopted in HEW, it can be used for ACK aggregation. However, consideration needs to be made to make it robust in case of error or missing ACK/BA (see figure 2).

The polled-ACK mechanism was added in 802.11ac for DL MU-MIMO, and it could be reused for DL OFDMA as well. Sequential-ACK procedures were considered in 11ac, but due to error recovery issues, none was adopted. Although polled-ACK is robust, it reduces the efficiency of the MU aggregation mechanism, particularly for short packets/bursts. While polled-ACK is suitable, it is not an

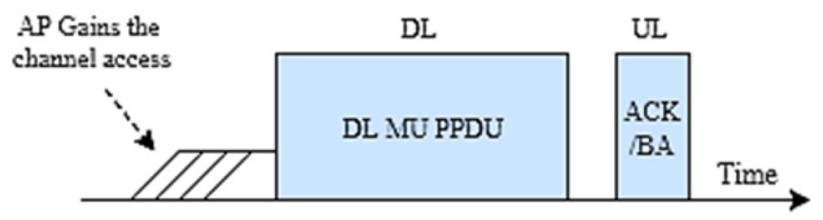

Figure 2. HE MU PPDU transmission with ACK/BA. efficient mechanism for short payloads, or to poll ACK from many STAs in case of DL OFDMA. Finding a new more efficient and yet robust ACK/BA aggregation improves the overall efficiency of MU aggregation. The UL OFDMA and UL MU might be used in 802.11ax, which are suitable candidates. Considering UL MU for ACK aggregation, although it is feasible and offers good error recovery, it is less favourable since it is likely to be an optional feature. UL OFDMA is likely to be considered for HEW if UL OFDMA can be used for ACK aggregation in UL direction [17].

Another alternative for ACK/BA aggregation in UL direction is to use CDMA-based signalling. CDMA-based signalling would also offer excellent trade-offs for ACK aggregation in UL direction, as well as for bandwidth/airtime request, which would be necessary for UL OFDMA and UL MU mechanisms. Use of CDMA-based signalling is also well known in OFDMA-based cellular technologies [18].

In LTE/WiMAX, there are dedicated common channels where STAs can put their request for UL airtime, where such resource is available to all STAs. Smartphone traffic measurements for the variety of applications such as YouTube, web browsing, Facebook, Skype, etc. are shown in figure 3. In this figure we can easily observe that packet sizes are mostly short, the number of packets per burst is mostly a few and burst inter-arrival time is mostly on the order of milliseconds [19]. For instance, traffic measurement on packet size reveals that packet size is $<66 \mathrm{~B}$ with $>75 \%$ chance for UL, and $<1500$ B with $40 \%$ chance for DL.

\subsection{HE sounding protocol}

Transmit beamforming and DL MU-MIMO need information about the channel state to calculate a steering matrix that is given to the transmit signal to improve reception at

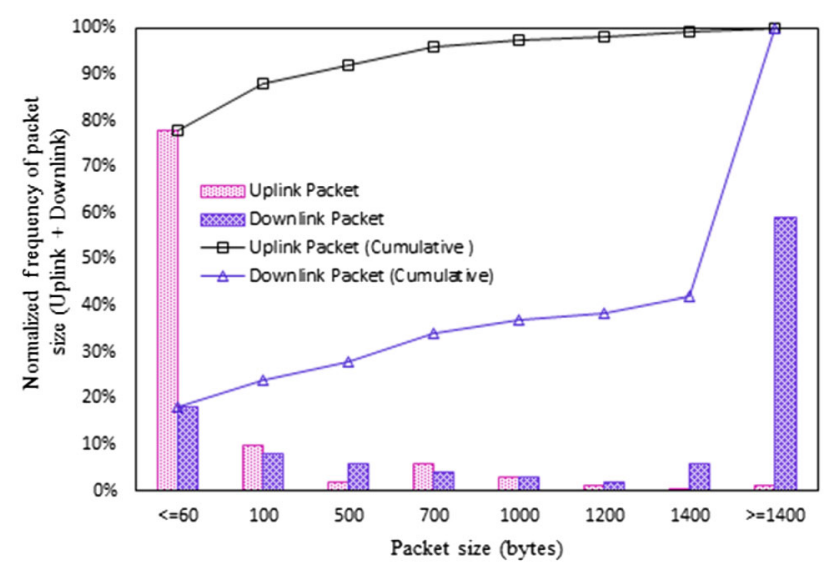

Figure 3. Distribution of uplink and downlink packet size for random web browsing. 


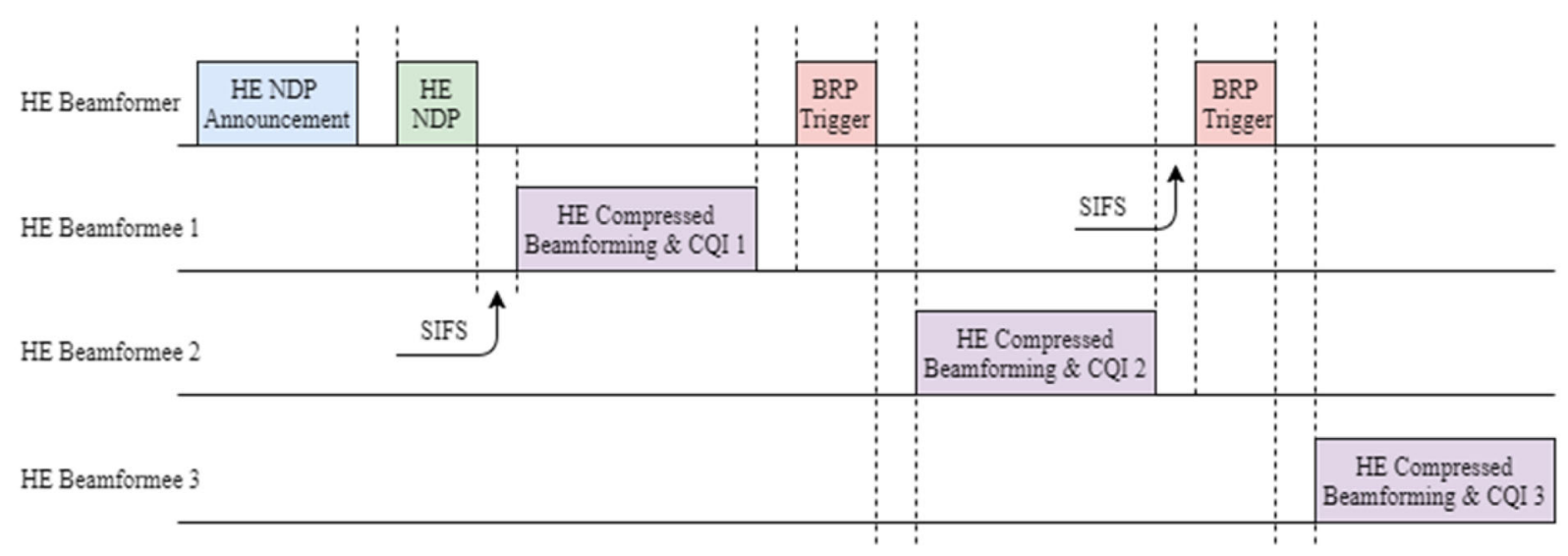

Figure 4. Transmit beamforming sounding protocol with more than one HE beamformee.

one or more receivers. Figure 4 shows that HE STAs use the HE sounding protocol to look for the channel state information. Just like the VHT sounding protocol, the HE sounding protocol uses an explicit feedback mechanism in which the HE beamformee estimates the channel using a training signal sent by the HE beamformer and forwards back a transformed assessment of the channel state. The HE beamformer uses this estimation to derive the steering matrix [2].

The HE beamformee provides an estimate information of the channel state in a HE compressed beamforming feedback. The HE compressed beamforming feedback is a HE compressed beamforming report field for SU-type feedback concatenation with the high-efficiency multi-user (HE MU) distinctive beamforming report field for MU-type feedback, and a channel quality information (CQI) only report field for CQI-type feedback [7]. The HE compressed beamforming feedback is taken in a single-HE compressed beamforming and CQI report frame if the induce frame is $\leq 11454$ octets in length. Otherwise, the HE beamforming feedback is segmented and each segment is taken in a HE compressed beamforming and CQI report frame. For CQItype feedback the HE compressed beamforming feedback is probably not segmented because induce MPDU size will always be $<11454$ octets.

\section{High-efficiency MAC}

To improve the system level effectiveness further and also for better use of spectrum resources in dense deployment scenarios, the 802.11ax standard uses an enhanced CCA that proposes to increase CCA-SD (signal detection)

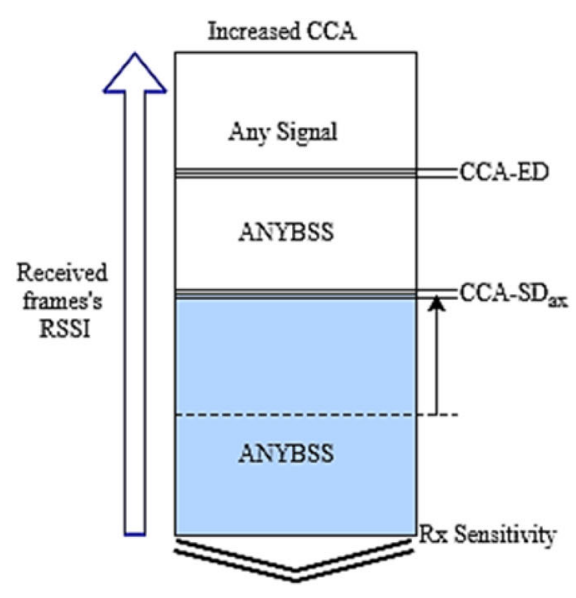

$\mathrm{CCA}-\mathrm{ED}=-62 \mathrm{~dB}$ for legacy
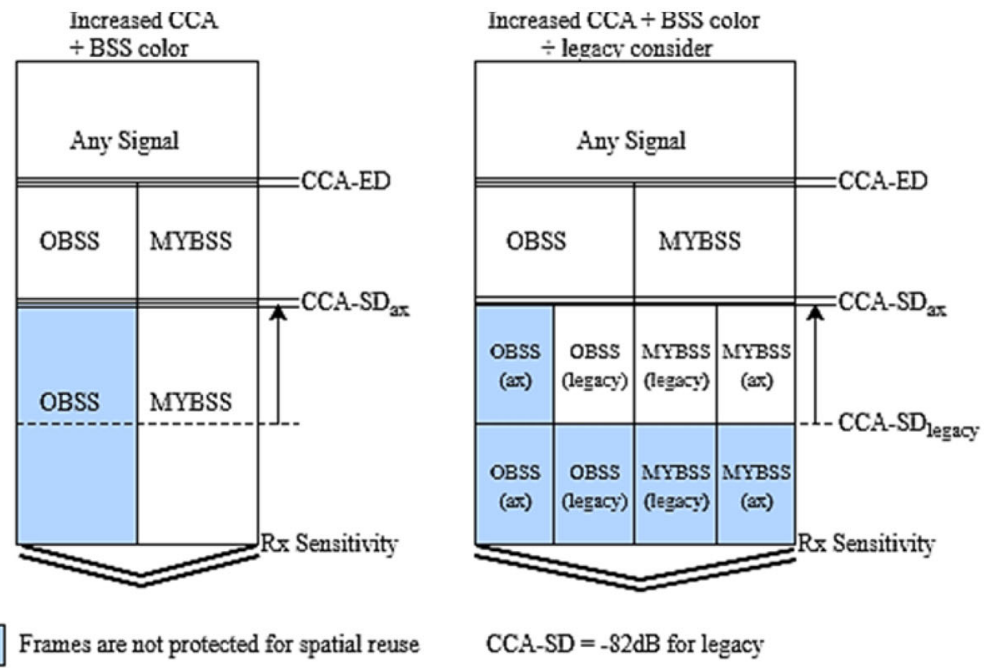

Figure 5. Using DSC (increased CCA) plus colour codes for spatial reuse. 


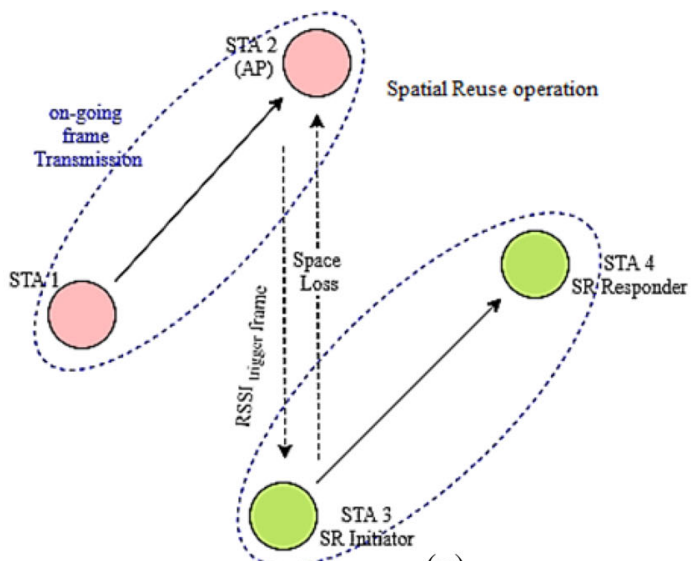

(a)

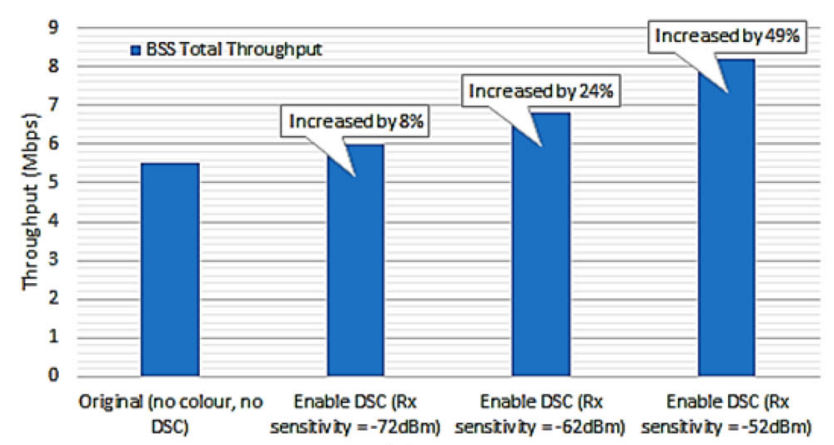

(c) Dynamic Sensitivity Control (DSC)

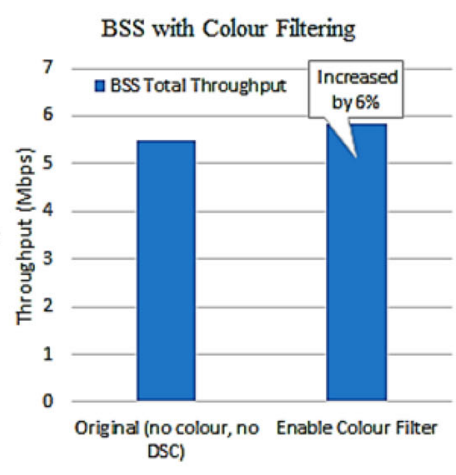

(b)

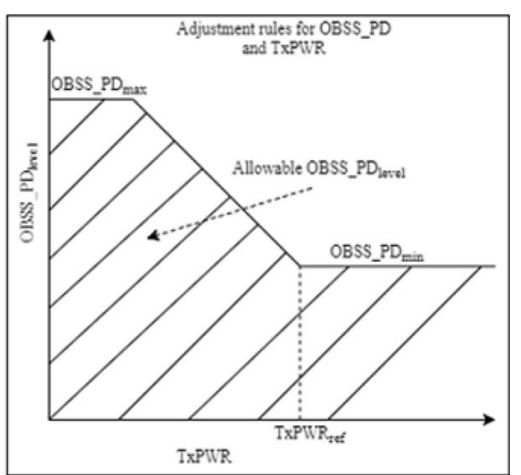

(e)

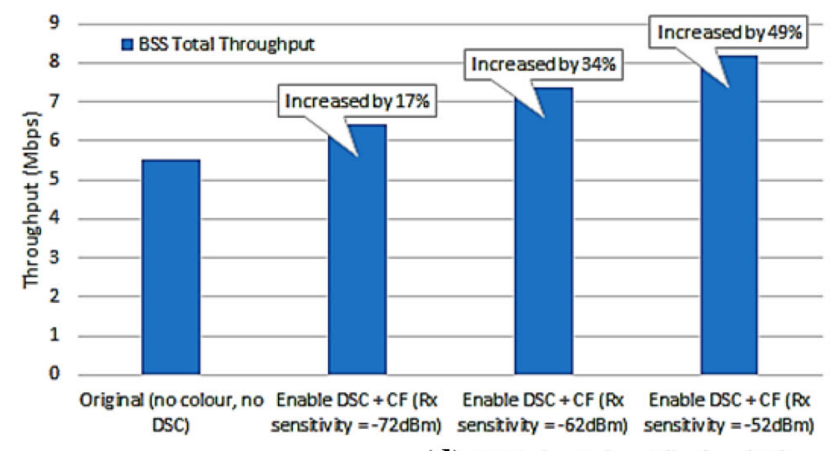

(d) DSC plus Colour Filtering (CF)

Figure 6. (a) Spatial reuse operation, (b)-(d) throughput comparison using BSS colour filter and DSC and (e) adjustment rule.

threshold to higher than $-82 \mathrm{dBm}$ for better SR. This new feature is known as DSC [20]. Using DSC, STAs can distinguish signals from OBSSs and make decisions on medium contention and interference management. As an alternative to carrier sense threshold (CSth), the transmit power control is adapted.

\subsection{Improving SR by increased CCA with BSS colouring}

To increase capacity in the dense environment, we need to improve frequency reuse between BSSs. BSS colouring was a mechanism introduced in 802.11ah to assign a different colour per BSS, which will be extending to dot11ax. New channel access behaviour will be awarded based on the colour detected. It identifies SR opportunities of acquiring knowledge from OBSSs using the BSS colour codes (see figure 5); SR operation is shown in figure $6 \mathrm{a}$.

Increased CCA: The 802.11ax AP/STA applies increased CCA-SD $\mathrm{Cx}_{\mathrm{ax}}$ level on any received frame [20]. However, the problems are the following: SR on MYBSS frame is not protected, reduction of DL/UL coverage due to the increased CCA and unfair to legacy STAs; it is not trivial to find the optimal CCA-SD $\mathrm{ax}_{\mathrm{ax}}$ level that satisfies all scenarios.

Increased CCA plus BSS colour: A frame received under $\mathrm{CCA}-\mathrm{SD}_{\mathrm{ax}}$ level is inspected for BSS colour (MYBSS frame), and OBSS frame is not protected. This case works only when there are no legacy STAs. HEW STA will require a longer time to inspect received frame's BSS colour while legacy STA will finish regular CCA in much shorter time on the same frame, which is a drawback of HEW STA. In this case also, OBSS legacy STA is unfair.

Increased CCA plus BSS colour plus legacy: A frame received under $\mathrm{CCA}-\mathrm{SD}_{\text {legacy }}$ and $\mathrm{CCA}-\mathrm{SD}_{\mathrm{ax}}$ is inspected for BSS colour, excluding $\mathrm{OBSS}_{\mathrm{ax}}$ frames. A frame received below $\mathrm{CCA}-\mathrm{SD}_{\text {legacy }}$ is also not protected. In this case, there are no significant problems like sacrificing MYBSS frame, legacy STA being unfairly treated and UL/ DL coverage reduction, but we need to work on SR for $\mathrm{OBSS}_{\mathrm{ax}}$ frames only. In future enhancement, we need to protect frames received under CCA-SD $\mathrm{D}_{\text {legacy }}$.

As an alternative to CSth, adapt the transmission power of the secondary user to minimize any harmful interference to the primary user. The duration of the SR opportunity is lower than the duration of the transmission from the primary user $[8,9,12,14,15,21]$. From the literature we find 
that there is not much improvement in BSS total throughput using only BSS colour filtering alone; it is shown in figure 6 b. However, we can easily observe that DSC provides more gain than colour filtering noted in figure 6c. Finally, when BSS colour filtering is used along with DSC, it can provide additional benefit when the offset of Rx sensitivity level is relatively small, which is acceptable for different application scenarios.

\subsection{Improving SR by $O A-C C A$}

OA-CCA is a technique used for SR. STA detects valid OBSS PPDUs and retrieves the SR field parameters that are an asset to SR. STA adjusts its transmit power to meet the SR operation before an SR attempt. In this situation, the HE STA may discard the OBSS PPDU if the SR condition is satisfied, i.e., the SR condition ensures that the receiving STA of the on-going OBSS transmission is not affected by the SR transmission, or else we just protect the receiver of the on-going frame exchange under TBD conditions. Two modes of SR operation are proposed by task group IEEE 802.11ax (TGax). First, OBSS_PD threshold is based on an adjustment of the transmit power and the OBSS_PD threshold without using spatial reuse parameter (SRP) in SR field, which allows simple semi-static implementation where an STA can set its OBSS_PD. For example, an STA can determine its TxPWR based on the path loss to the intended receiver (in our case STA2) and environment and raise the OBSS_PD threshold. Second is OA-CCA; this mode is based on an SR field in HE-SIG-A and per link detection. First, we need to adjust the transmit power based on the specific information of path losses and acceptance receiver interference level to protect the receiver of the on-going transmission, based on the specific link detected like one PPDU at a time, which allows gathering of per BSS colour information to operate on a per colour and per SRP basis; this is more accurate and specific to dense deployment $[8,9,12]$.

OA-CCA for Trigger frame and uplink MU: Trigger frame from AP (in our case STA2) for uplink multi-user carries SRP, OBSS colour and the uplink duration; meanwhile uplink STA1 copies SRP of the trigger frame (RSSI $\left.I_{\text {trigger frame }}\right)$ into the SR field. STA3, which is denoted as an SR initiator, can initiate an SR transmission during the uplink PPDU duration after receiving the SRP. It should be noted that the STA can operate at the legacy CCA level without employing a higher OBSS_PD level. The adjustment rule is illustrated in figure $6 \mathrm{e}$.

Mathematical analysis of the SR operation: STA1, which is also noted as UL STA, transmits to STA2 and STA1's transmission is detected by STA3 as it is above the baseline CCA level; STA3 identifies OBSS transmission and tries to initiate an SR transmission to STA4. For STA3 not to interfere with AP reception named as STA2, here we have the condition
TxPWR $_{\mathrm{STA} 3}$ - Space Loss $<$ Acceptable Receiver

Interference Level STA2(AP) $_{2}$

where Space Loss $=\mathrm{TxPWR}_{\mathrm{STA} 2(\mathrm{AP})}$ - RSSI trigger frame@STA3.

Define SR parameter (SRP) as follows:

$\mathrm{SRP}=\mathrm{TxPWR}_{\mathrm{STA} 2(\mathrm{AP})}$

+ Acceptable Receiver Interference Level STA2(AP) $_{\text {. }}$.

The required transmit power back - off is simplified

to $\mathrm{TxPWR}_{\mathrm{STA} 3}<S R P-\mathrm{RSSI}_{\text {triggerframe@STA3 }}$.

All power levels are normalized to that of $20 \mathrm{MHz}$ :

power $-10 \times \log \left(\mathrm{BW} / \mathrm{BW}_{20 \mathrm{MHz}}\right)$.

\subsection{Access efficiency (MAC efficiency)}

The efficiency of WLAN channel use can be as low as $10 \%$ in certain conditions, which means that successful WLAN data transmission is lessened to $10 \%$ while $\sim 90 \%$ of the channel is used for control, management frames and lost transmissions (collisions). HEW must permanently greet this problem [7]. An access efficiency requirement for HEW would provide significant improvements for overloaded hotspots. Access efficiency is calculated by dividing the BSS average total throughput by the BSS throughput time-averaged data rate. This metric provides the unit-less percentage of time over which the channel is occupied by data packets that are successfully transmitted and received. Control frames, management frames and collision frames are excluded from access efficiency calculation. The HEW amendment provides a mechanism to increase BSS channel access efficiency in overloaded channel scenarios with many associated STAs. The HEW amendment includes at least one mode of operation capable of achieving $60 \%$ or greater access efficiency when compared with scenarios providing 20-25\% access efficiency [3, 4, 8, 9] when using existing IEEE 802.11 standard and its amendments operating in the same band in the same deployments with the same number of STAs and equal traffic loading. Figure 7 shows that the HEW requires both MAC efficiency and PHY data rate to achieve the functional requirements.

\subsection{Enhanced TWT operation}

TWTs allow STAs to maintain activity in the BSS by scheduling STAs to operate at different times to minimize contention between STAs and to reduce the required amount of time that an STA in power save (PS) mode needs 


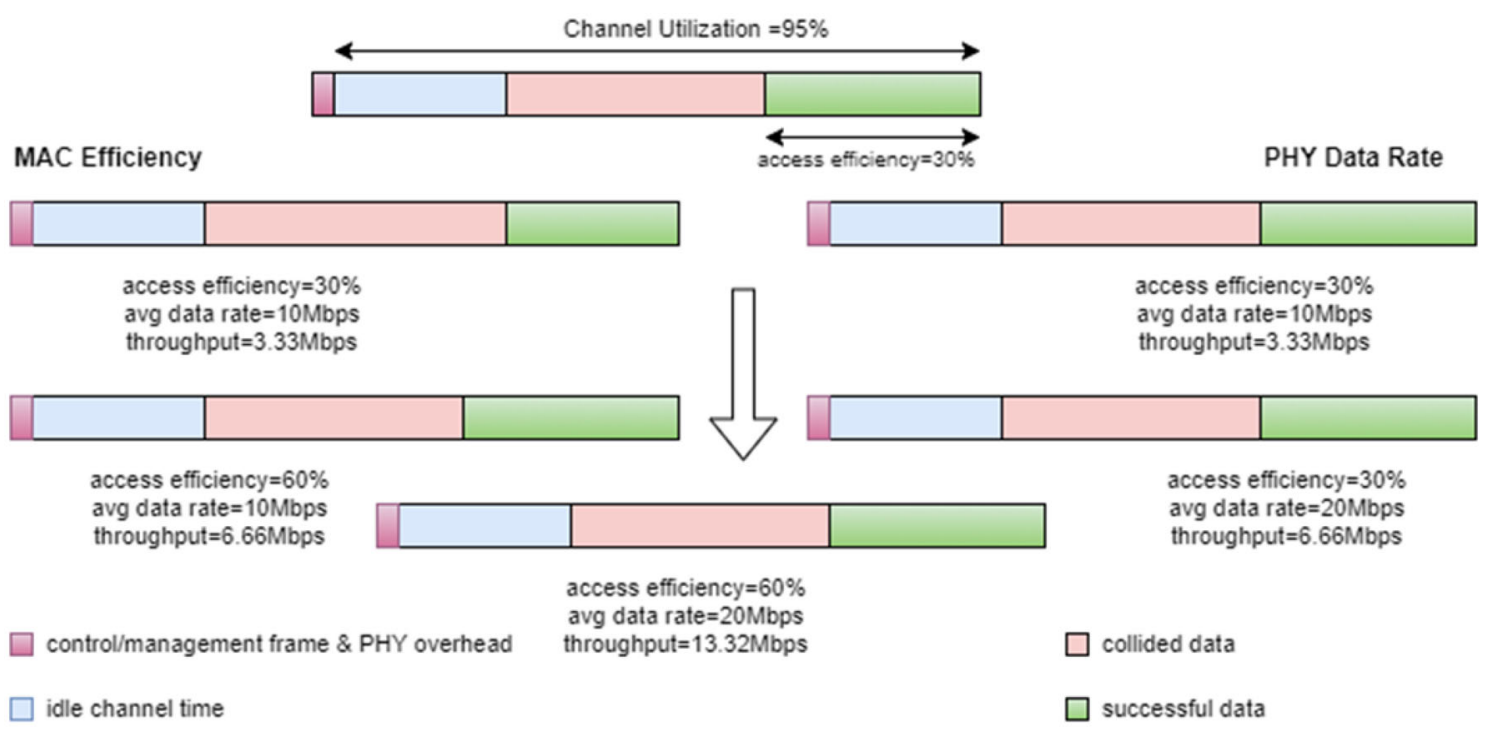

Figure 7. Throughput enhancement using MAC efficiency plus PHY data rate.

to be awake. The new proposal made by TGax to enhance TWT operation is a three-way approach. First, signalling a schedule when an STA is not able to transmit or receive, second, TWT flow set-up for power savings and constrained periods, and third, TWT set-up response. The first approach, a TWT requesting STA, requires listing the repeating times, i.e., constrained periods when it is not able to transmit or receive. If needed, the TWT receiving STA should use this information to select the TWT SP times, i.e., select the times when the requesting STA can transmit and receive. In the second approach, one TWT set-up frame requests a single-TWT agreement. To set up a TWT flow for power save and TWT flow to avoid operation at times when STA is not available (see figure 8), the STA should transmit two TWT set-up request frames: one TWT set-up frame requesting a TWT flow for power save and one TWT set-up frame requesting a TWT flow for constrained periods to provide information on the times when STA is not available. The two set-up frames may be aggregated to the same PPDU and transmitted at the same time. In the third approach, the AP may accept or reject the TWT- constrained periods agreement, i.e., schedule when the requesting STA is not available. If accepted, the requesting STA shall update the constrained periods agreement if its availability changes. When constrained periods and TWT agreement for power save are transmitted in the same PPDU, the AP may accept only TWT agreement for power save, but the STA shall not update its availability information to the AP. The AP may propose alternative parameters for TWT agreement for power save. The TWT parameter set on constrained periods, i.e., when STA is not available, helps in selecting a suitable schedule.

\section{Throughput analysis}

In this section, we study the single-AP throughput analysis for four user downlink scenarios using HE MU PPDU frame format that supports the three MU-MIMO, OFDMA and combination of both configurations. The HE MU configuration is used to configure the transmission (Tx) of a HE MU packet. The HE MU PPDU format shown in the

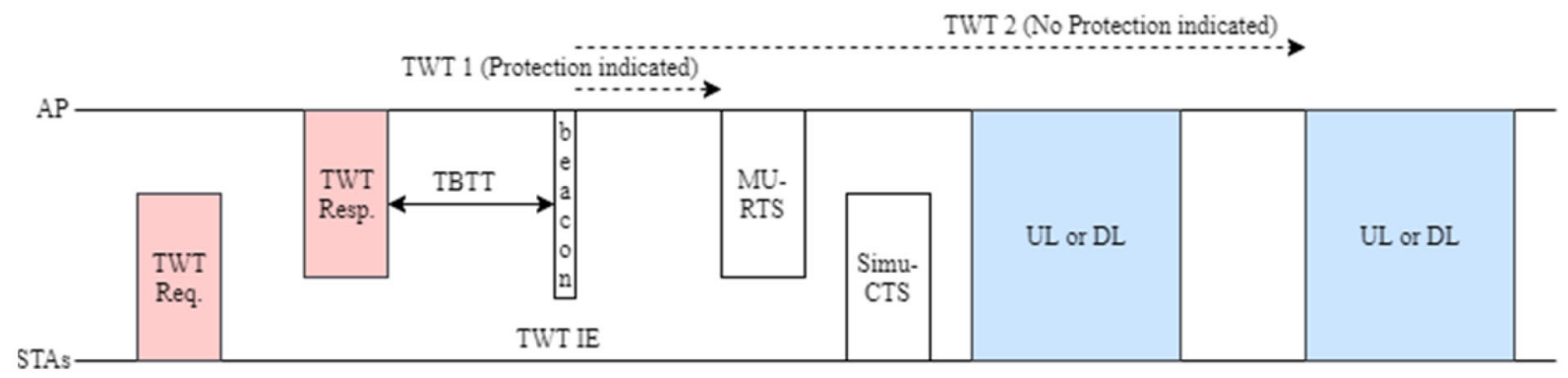

Figure 8. Enhanced TWT operation. 


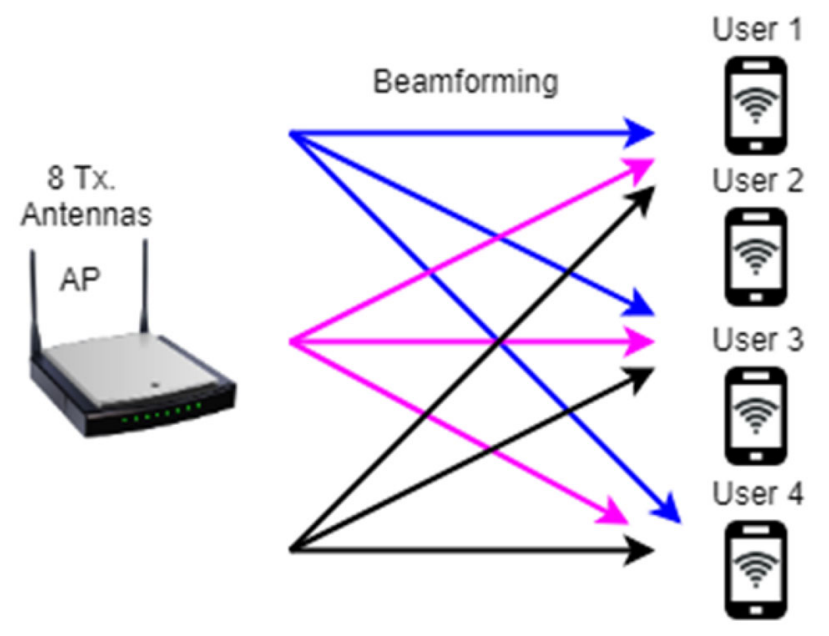

Figure 9. Downlink scenario (one to many) using high-efficiency WiFi.

PHY section includes the RU allocation for multiple WLAN STAs, along with the configuration details for each user such as the modulation and coding schemes (MCS), number of spatial streams and STA ID. The indoor MIMO channel along with AWGN is modelled between AP and each STA shown in figure 9. Here we assume that a maximum of two collisions are possible; therefore, our exponential back-off (EBF) slots are utmost equal to 3 [9, 22]. In these configurations we consider two possible schemes by varying the payload size (APEP Length) and operating SNR for three distinct AP transmissions with a burst of 10 packets. (1) MU-MIMO configuration, which is made up of a single 242-tone RU using four users. Each user offers one space time stream. (2) OFDMA configuration, which is made up of four 52-tone RUs, each having one user and each user offers two space-time streams. (3) Mixed configuration (MU-MIMO plus OFDMA), which is made up of one 106-tone RU shared by two MU-MIMO users, and each offers one space-time stream. Two space-time streams are offered by each OFDMA user, which is made up of two 52-tone RUs, each with one user.

Allocation index or contention index for all configurations is listed in table 3. The RU allocation index for each $20-\mathrm{MHz}$ subchannel is specified as a row vector of integers between 0 and 223, both inclusive. The allocation index
Table 4. Simulation parameters for AP throughput analysis.

\begin{tabular}{lc}
\hline Parameters & Value \\
\hline Sample rate & 20000000 \\
Delay profile & Model-D \\
Channel bandwidth & $20 \mathrm{MHz}$ \\
Carrier frequency & $5.25 \mathrm{GHz}$ \\
Transmitter-receiver distance & $10 \mathrm{~m}$ \\
Antenna spacing at both Tx and Rx side & $0.5 \lambda$
\end{tabular}

Fluorescence effect

MCS

Number of packets considered for each path loss

AP transmit power

STA noise floor

Idle time between the packets

Number of users

Number of transmit antennas (AP side)

Number of receive antennas for each STA

Channel coding

HELTF type

TXOP duration

Guard interval

Transmission direction

FFT size

True or turn-on

4

10

$30 \mathrm{dBm}$

$-89.9 \mathrm{dBm}$

$20 \mu \mathrm{s}$

4

8

2

LDPC

4

127

$0.8 \mu$ s (Base)

Downlink 256

defines the number and sizes of RUs, and the number of users assigned to each RU. The length of allocation index must be 1, 2, 4 or 8 , defining the assignment for each $20-\mathrm{MHz}$ subchannel in a 20-, 40-, $80-$ or $160-\mathrm{MHz}$ channel bandwidth, or for a full band allocation with a single RU. The $20-\mathrm{MHz}$ channel bandwidth is used for all transmissions [13, 23]. Some other variables like MCS and number of receive antennas for each user will be fixed in all configurations; in our simulation we set MCS equal to 4, number of transmit antennas used in AP side to 8 and number of receive antennas for each STA equal to 2. Here we use the latest version of MATLAB 2018a, which supports HEW. The variables or parameters used in the WLAN system toolbox for these configurations are noted in table 4 .

As explained in section 4.2, the 802.11ax AP decrees the STAs to modify their power increase or decrease based on the target received signal strength indicator (RSSI) at the AP side. The STA first measures free space path loss using Eq. (4.2), i.e., subtracting measured RSSI (at STA) from AP transmit power (encoded inside the packet). The STA

Table 3. Resource allocation table for four-user AP throughput analysis.

Allocation index

0 (reference purpose)

24-31 (23 + number of users) (mixed mode transmission)

112 (OFDMA transmission)

192-199 (191 + number of users) (MU-MIMO transmission)
20-MHz subchannel resource unit (RU) assignment

\begin{tabular}{|c|c|c|c|c|c|c|c|c|}
\hline 26 & 26 & 26 & 26 & 26 & 26 & 26 & 26 & 26 \\
\hline \multicolumn{4}{|c|}{106 (1-8 users) } & - & \multicolumn{2}{|r|}{52} & \multicolumn{2}{|r|}{52} \\
\hline & 2 & \multicolumn{2}{|c|}{52} & - & & 52 & & 52 \\
\hline \multicolumn{9}{|c|}{242 (1-8 users) } \\
\hline
\end{tabular}


Table 5. Throughput and access efficiency using MCS 4.

\begin{tabular}{|c|c|c|c|c|}
\hline \multirow[b]{3}{*}{$\begin{array}{l}\text { Path } \\
\text { loss } \\
(\mathrm{dB})\end{array}$} & \multicolumn{4}{|c|}{ Payload size $=1508 \mathrm{~B}, \mathrm{PHY}$ rate $=52 \mathrm{Mbps} / \mathrm{STA}$} \\
\hline & \multicolumn{2}{|c|}{ OFDMA configuration } & \multicolumn{2}{|c|}{ MU-MIMO configuration } \\
\hline & $\begin{array}{c}\text { Throughput } \\
\text { (Mbps) }\end{array}$ & $\begin{array}{c}\text { MAC } \\
\text { efficiency } \\
(\%)\end{array}$ & $\begin{array}{c}\text { Throughput } \\
\text { (Mbps) }\end{array}$ & $\begin{array}{c}\text { MAC } \\
\text { efficiency } \\
(\%)\end{array}$ \\
\hline 96 & 71.6 & $84.44 *$ & 130 & 62.5 \\
\hline 98 & 71.6 & $84.44 *$ & 130 & 62.5 \\
\hline 100 & 71.6 & $84.44 *$ & 130 & 62.5 \\
\hline 102 & 53.7 & $63.33 *$ & 65 & 31.25 \\
\hline 104 & 35.8 & $42.22 *$ & 0 & 0 \\
\hline 106 & 26.8 & $31.6^{*}$ & 0 & 0 \\
\hline
\end{tabular}

then transmits a signal with power equal to the target RSSI plus estimated path loss. STAs closer to the AP transmit less power while STAs farther away transmit more power to overcome the higher path loss. The RSSI values provisions by the vendors and chipset makers like Aruba HPE, Cisco and so on, and not by IEEE standard. Generally, $-50 \mathrm{dBm}$ RSSI value provides $100 \%$ quality, also called as excellent signal strength. However, once RSSI value decreases to the noise floor, for example, $-100 \mathrm{dBm}$, quality will be $0 \%$ (table 5); this leads to data corruption and requires a more significant number of retransmissions, adversely affecting throughput and latency. Table 6 shows the predefined values of RSSI, which define the path loss and SNR.

SNR is not a ratio but the difference in decibels between the received signal and the background noise level (noise floor). For example, if a radio (client device) receives a signal of $-67 \mathrm{dBm}$ and the noise floor is measured at $-90 \mathrm{dBm}$, the SNR is $23 \mathrm{~dB}$. In this scenario, SNR will vary between 24 and $14 \mathrm{~dB}$ when path loss varies from 96 to $106 \mathrm{~dB}$, and this is because RSSI value ranges from -66 to $-76 \mathrm{dBm}$. The $-67 \mathrm{dBm}$ signal strength is very good for voice over $\mathrm{Wi}-\mathrm{Fi}$ and non-HD streaming video applications that satisfy functional requirements observed in figures 10 and 11 having high SNR value of $23 \mathrm{~dB}$, and $97 \mathrm{~dB}$ low path loss.

Scheme 1: In this scheme, the APEP length is set to 1508 $\mathrm{B}$ for all users in all configurations, which is considered as transmission of large size packet under the conditions of AP throughput analysis. Eight transmit antennas (AP side) and two receive antennas (STA side) are used in this scheme $(8 \times 2)$. The result is shown in figure 10 ; in high SNR region (low path loss), MU-MIMO configuration gives better AP throughput value of $130 \mathrm{Mbps}$ when compared with OFDMA configuration, which achieves 71.6 Mbps. The packet duration of the MU-MIMO configuration is approximately $50 \%$ that of the OFDMA configuration, which gives throughput gain. As the SNR decreases (high path loss), OFDMA configuration gets more effective with the help of transmit beamforming. The
Table 6. Relationship among path loss, SNR and RSSI.

\begin{tabular}{|c|c|c|c|}
\hline $\begin{array}{l}\text { RSSI } \\
(\mathrm{dBm})\end{array}$ & $\begin{array}{l}\text { Path } \\
\text { loss } \\
\text { (dB) }\end{array}$ & $\begin{array}{l}\text { SNR } \\
(\mathrm{dB})\end{array}$ & Informative with respect to RSSI \\
\hline-30 & 60 & 60 & $\begin{array}{l}\text { Perfect signal strength, not } \\
\text { acceptable in the real world; the } \\
\text { client can be touching feet from AP } \\
\text { to achieve this }\end{array}$ \\
\hline-50 & 80 & 40 & $\begin{array}{l}\text { Anything down to this level can be } \\
\text { considered an excellent signal } \\
\text { strength }\end{array}$ \\
\hline-60 & 90 & 30 & Good and reliable signal \\
\hline-67 & 97 & 23 & $\begin{array}{c}\text { Minimum signal strength for VoWiFi } \\
\text { and non-HD streaming video } \\
\text { applications that are very reliable, } \\
\text { timely packet delivery }\end{array}$ \\
\hline-70 & 100 & 20 & $\begin{array}{c}\text { Minimum signal strength for reliable } \\
\text { packet delivery; e-mail and web } \\
\text { applications }\end{array}$ \\
\hline-80 & 110 & 10 & $\begin{array}{l}\text { Minimum signal strength for basic } \\
\text { connectivity; packet delivery may } \\
\text { be unreliable }\end{array}$ \\
\hline-90 & 120 & 0 & $\begin{array}{l}\text { Equalling or drowning in the noise } \\
\text { floor; any functionality is highly } \\
\text { implausible }\end{array}$ \\
\hline
\end{tabular}

packet durations of OFDMA and mixed configuration are relatively same [24].

Scheme 2: In this scheme, the APEP length is set to $36 \mathrm{~B}$ for all users in all configurations, which is considered as transmission of small size packet under the conditions of AP throughput analysis. The $8 \times 2$ matrix is the same as in scheme 1 . The result is shown in figure 11; for small packet size, Tx OFDMA and mixed configuration provide better AP throughput irrespective of high/low-SNR region. MUMIMO configuration suits only at high SNR (low path loss). The four-user cumulative throughput of OFDMA configuration in high SNR region is $8.8 \mathrm{Mbps}$ and it maintains half of the value till the end; because of this behaviour, OFDMA configuration outperforms in the entire SNR region [24].

Special case: This scheme belongs to performance evaluation of 5th percentile STAs usually present in the high-path-loss or low-SNR region, to meet functional requirements as discussed in section 2. Here the APEP length is set to $1000 \mathrm{~B}$ for all MU-MIMO users, and $100 \mathrm{~B}$ for all OFDMA users in all configurations. Six transmit antennas (AP side) and two receive antennas (STA side) are used in this scheme $(6 \times 2)$. From figure 12 , it is easily understood that solitary MU-MIMO configuration (802.11ac) cannot resist the high path loss; however, in other configurations like OFDMA and mixed mode (802.11ax), throughput is consistently maintained till the end, which means even in the 5 th percentile, STAs can get desirable throughput as per norms $(4 \times)$. 


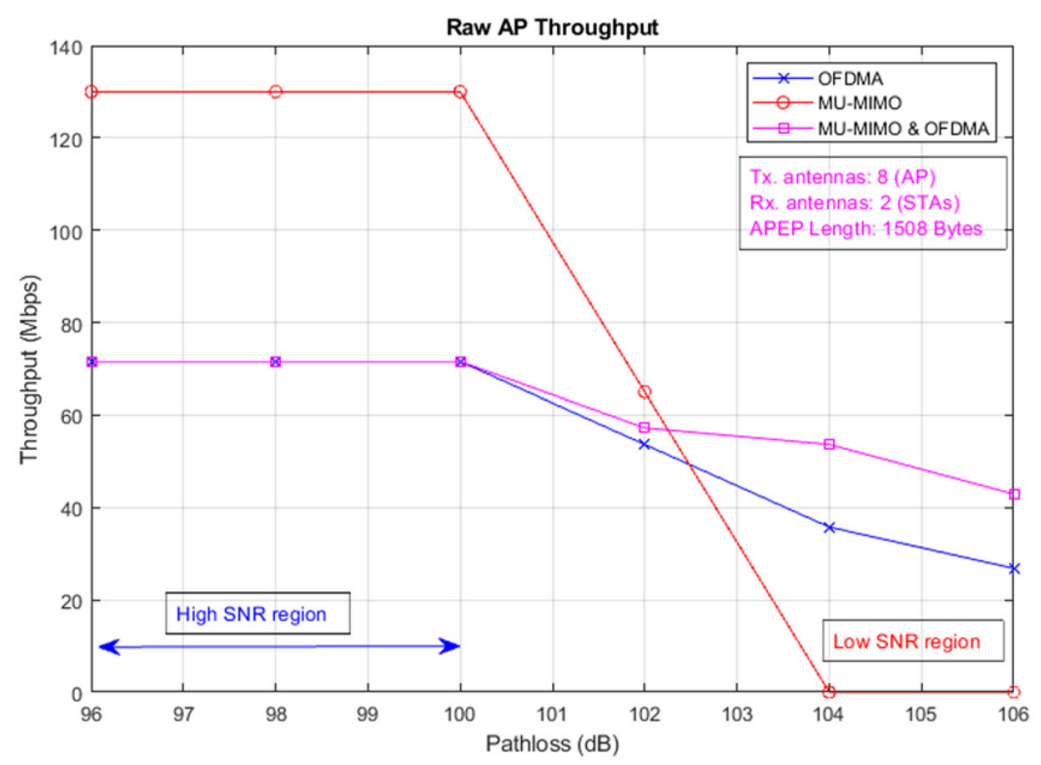

Figure 10. Scheme 1-performance of large size packet transmission.

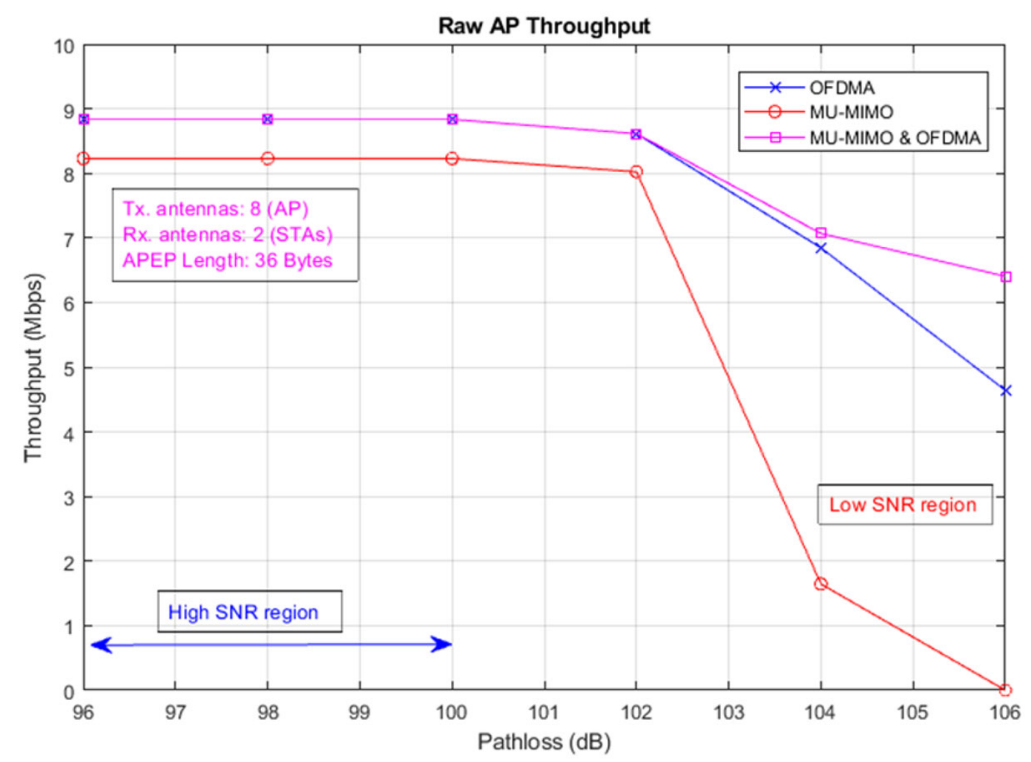

Figure 11. Scheme 2-performance of small size packet transmission.

As explained in the access efficiency section, $60 \%$ efficiency is easily obtainable in 802.11ax for dense environments as shown in table 5; also the calculation efficiency using PHY rate for each user or STA is $52 \mathrm{Mbps}$. For low path loss, $62.5 \%$ efficiency is obtained in MU-MIMO configuration and OFDMA configuration can maintain the average efficiency of $26.5 \%$ till the end. Calculation of efficiency performed using Eqs. (5.1) and (5.2) defines the MAC throughput as the ratio of total MAC payload (bits) to the time consumed for transmitting total MAC payload (s) [11, 25]. For example, at $96 \mathrm{~dB}$ path loss, OFDMA configuration can send a payload of 1508 bytes for 4 users with a burst of 10 packets equal to $482.56 \mathrm{kB}$. Hence, the total time required to transmit $482.56 \mathrm{kB}$ is $6.74 \mathrm{~ms}$. This time duration is very much acceptable to satisfy the functional requirements in dense scenarios using high efficiency Wi-Fi or wireless.

MAC throughput $=$ total MAC payload for each path loss(B) time consumed for transmitting total MAC payload(s) 


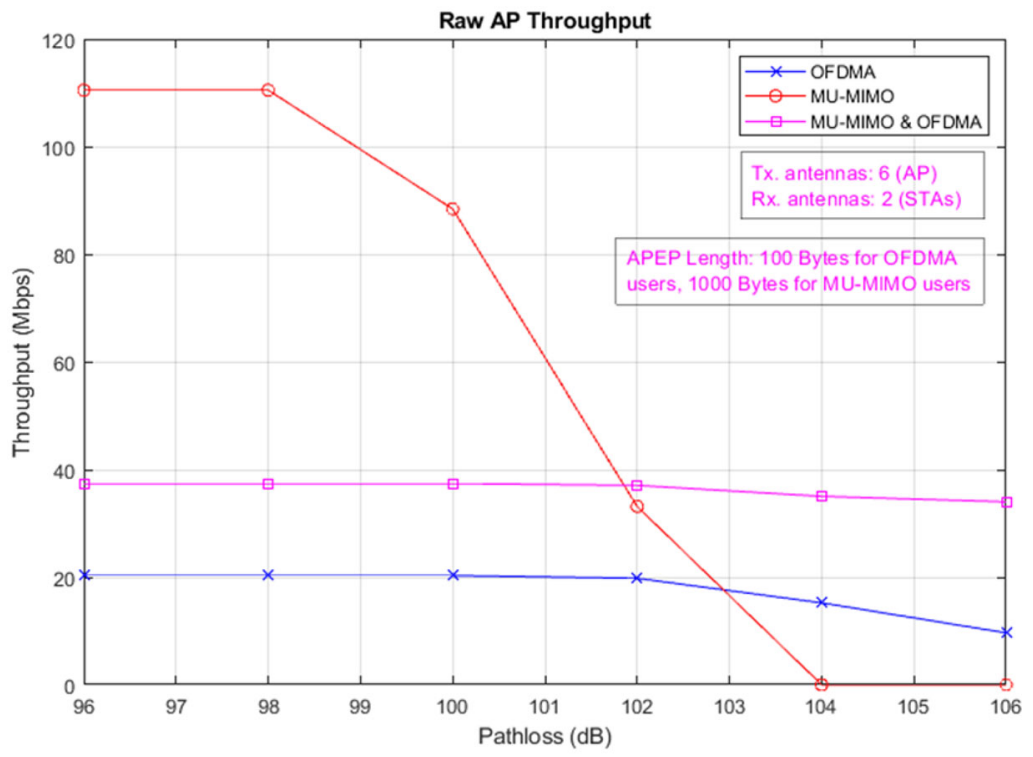

Figure 12. Performance of 5th percentile STAs.

$$
\text { MAC efficiency }=\frac{\text { MAC throughput }}{\text { PHY rate }} .
$$

\section{Conclusion}

In this article, we briefly reviewed a few technological building blocks of IEEE 802.11ax-2019 (HEW) amendment being designed to increase capacity in dense environments. Interactive video transmission latency requirements provide $\mathrm{QoS}$ in overloaded APs for AC_VI. Trigger-based OFDMA transmissions and uplink MU-MIMO are the most disruptive improvements in high-efficiency PHY from previous approved 802.11n/ac amendments. HEW may solely outperform VHT WLANs in scenarios with many STAs associated with a single AP, as shown in table 5. High-efficiency MAC works on improving SR by DSC; BSS colouring and OA-CCA seem very promising approaches to reduce the exposed node problem in high-density WLANs, and hence improve the area throughout. The throughput analysis result shows that for large packets, MUMIMO is the most efficient in high-SNR region. OFDMA is the most efficient over the entire SNR range for a small packet. In future, WLAN scenarios will have coexistence with unlicensed LTE; to avoid this we need to combine HEW and IEEE 802.11ad/ay WLANs, and many other legacy features.

\section{References}

[1] Ramaiyan V 2009 Topics in modeling, analysis and optimization of wireless networks. Ph.D. thesis, Indian Institute of Science, Bengaluru
[2] Perahia E and Stacey R 2013 Next generation wireless LANs: 802.11n and 802.11ac, 2nd ed. Cambridge University Press, India

[3] Palit R, Naik K and Singh A 2012 Anatomy of WiFi access traffic of smartphones and implications for energy saving techniques. International Journal of Energy, Information and Communication 3(1): 1-16

[4] Omar H A, Abboud K, Cheng N, Malekshan K R, Gamage A T and Zhuang W 2016 A survey on high efficiency wireless local area networks: next generation WiFi. IEEE Communications Surveys \& Tutorials 18(4): 2315-2344

[5] Sharon O and Alpert Y 2017 Single user MAC level throughput comparison: IEEE 802.11ax vs. IEEE 802.11ac. Wireless Sensor Network 9: 166-177

[6] Vijay B T and Malarkodi B 2019 MAC improvements for very high throughput WLANs. International Journal of Communication Networks and Distributed Systems 22(1): 74-97

[7] IEEE P802.11 - Task Group ax 2016 Group information update: status of project IEEE 802.11ax. High Efficiency Wireless LAN Task Group

[8] Afaqui M S, Garcia-Villegas E and Lopez-Aguilera E 2017 IEEE 802.11ax: challenges and requirements for future high efficiency WiFi. IEEE Wireless Communications 24(3): 130-137

[9] Yang D X, Guo Y and Aboul-Magd O 2017 802.11ax: the coming new WLAN system with more than $4 \times$ MAC throughput enhancement. In: Proceedings of the 86th IEEE Vehicular Technology Conference (VTC-Fall), pp. 1-5

[10] Bellalta B 2016 IEEE 802.11ax: High-efficiency WLANS. IEEE Wireless Communications 23(1): 38-46

[11] IEEE Standard for Information Technology 2016 Telecommunications and information exchange between systems local and metropolitan area networks—specific requirements part 11: Wireless LAN medium access control (MAC) and physical layer (PHY) specifications. IEEE Std 802.11-2016 (Revision of IEEE Std 802.11-2012) 
[12] Deng D J, Lin Y P, Yang X, Zhu J, Li Y B, Luo J and Chen K C 2017 IEEE 802.11ax: Highly efficient WLANs for intelligent information infrastructure. IEEE Communications Magazine 55(12): 52-59

[13] Ali R, Kim S W, Kim B S and Park Y 2018 Design of MAC layer resource allocation schemes for IEEE 802.11ax: future directions. IETE Technical Review 35(1): 28-52

[14] Cheng N and Shen X S 2017 Next-generation high-efficiency WLAN. In: Proceedings of the $5 G$ Mobile Communications. Cham: Springer, pp. 651-675

[15] Zhang Y, Li B, Yang M, Yan Z and Zuo X 2017 An OFDMA-based joint reservation and cooperation MAC protocol for the next generation WLAN. Wireless Networks, https://doi.org/10.1007/s11276-017-1567-1, pp. 1-15

[16] Zhou H, Li B, Yan Z and Yang M 2017 A channel bonding based QoS-aware OFDMA MAC protocol for the next generation WLAN. Mobile Networks and Applications 22(1): 19-29

[17] Kosek-Szott K 2018 Improving DL MU-MIMO performance in IEEE 802.11ac networks through decoupled scheduling. Wireless Networks 24(8): 3113-3127

[18] Bellalta B and Kosek-Szott K 2017 AP-initiated multi-user transmissions in IEEE 802.11ax WLANs. arXiv: 1702.05397

[19] Khorov E, Kiryanov A, Krotov A, Gallo P, Garlisi D, Tinnirello I and Kiryanov A 2016 Joint usage of dynamic sensitivity control and time division multiple access in dense 802.11ax networks. In: Proceedings of the International Workshop on Multiple Access Communications. Cham: Springer, pp. 57-71

[20] Faridi A, Bellalta B and Checco A 2017 Analysis of dynamic channel bonding in dense networks of WLANs. IEEE Transactions on Mobile Computing 16(8): 2118-2131

[21] Yang B, Li B, Yan Z and Yang M 2018 A channel reservation based cooperative multi-channel MAC protocol for the next generation WLAN. Wireless Networks 24(2): 627-646

[22] Deng D J, Lien S Y, Lee J and Chen K C 2016 On quality-ofservice provisioning in IEEE 802.11ax WLANs. IEEE Access 4: 6086-6104

[23] Aijaz A and Kulkarni P 2017 Simultaneous transmit and receive operation in next generation IEEE 802.11 WLANs: a MAC protocol design approach. IEEE Wireless Communications 24(6): 128-135

[24] IEEE 802.11-15/0333r0 2015 Throughput comparison of some multi-user schemes in 802.11ax. IEEE TGax

[25] Khorov E, Loginov V and Lyakhov A 2016 Several EDCA parameter sets for improving channel access in IEEE 802.11ax networks. In: Proceedings of the International Symposium on Wireless Communication Systems (ISWCS), Poznan, pp. 419-423 